\title{
ON GROUPS ALL OF WHOSE 2-BLOCKS HAVE THE HIGHEST DEFECTS
}

\author{
KOICHIRO HARADA
}

\section{Introduction}

If $G$ is a finite group and $p$ a fixed prime number, the irreducible representations of $G$ are distributed into disjoint systems, the $p$-blocks. These blocks have been investigated especially by $R$. Brauer. In this note we are concerned with the problem: What is the structure of $G$ which has only one $p$-block? Or more generally, what is the structure of $G$ all of whose $p$-blocks have the highest defects?

It is well known that if a finite group $G$ has a non-trivial normal $p$-subgroup $N$ such that the centralizer of $N$ is contained in $N$, then $G$ has only one $p$-block (see Curtis-Reiner [3], p. 634, Ex. 1). Therefore it is interesting to consider the converse of this theorem. For $p$-solvable groups, the converse is true. Indeed, since $O_{p^{\prime}}(G)$ is a intersection of kernels of all the characters contained in the first $p$-block of $G$ (R. Brauer [1]), if a $p$-solvable group $G$ has only one $p$-block, then $O_{p}(G)=1$. Therefore Lemma 1.2.3 of P. Hall-G. Higman [6] shows that $C_{G}\left(O_{p}(G)\right) \subset O_{p}(G)$. Hence the converse holds for $p$-solvable groups. In general the converse is not true. In fact, the Mathieu group of degree 24 and that of degree 22 have only one 2-block (for the character tables of the Mathieu groups, see G. Forbenius [5], N. Burgoyne and P. Fong [2]). The purpose of this note is to prove the following theorem.

Theorem 1. Let $G$ be a finite group with an abelian Sylow 2-subgroup $S$. Let $|G|=2^{a} \cdot g^{\prime},\left(2, g^{\prime}\right)=1$. Then every 2-block of $G$ has defect $a$ if and only if $S$ is normal in $G$.

Corollary. If a finite group $G$ with an abelian Sylow 2-subgoup $S$ has only one 2-block, then $S$ is normal in $G$.

Received July 17, 1967. 


\section{Proof of Theorem}

We shall first prove two lemmas.

Lemma 1. Let $G$ be a finite p-solvable group, with an abelian Sylow p-subgroup S. If every p-block of $G$ has the highest defect, then $S$ is normal in $G$.

Proof. Let $|G|=p^{a} g^{\prime}, \quad\left(p, g^{\prime}\right)=1$. We shall apply a result of P. Fong [4]. Since $S$ is abelian, by Theorem $(3 C)$ of [4] every irreducible character has height 0 . Since every $p$-block of $G$ has defect a, every irreducible character of $G$ has degree prime to $p$. By Lemma $(3 D)$ of [4], $S$ is normal in $G$.

Lemma 2. If every $p$-block of a finite group $G$ with an abelian Sylow $p$-subgroup has the highest defect, then the centralizer $C_{G}(x)$ of any p-element $x$ of $G$ has the same property as $G$.

Proof. Suppose that $H=C_{G}(x)$ has a block $B$ of defect $d<a$, where $|G|=p^{a} \cdot g^{\prime},\left(p, g^{\prime}\right)=1$. Let $D$ be a defect group of $B$ (in $H$ ). Then by a theorem of $\mathrm{R}$. Brauer $C_{H}(D) \cdot D=C_{H}(D)$ has a block of defect $d$. Since $x \in D, C_{H}(D)=C_{G}(D)$. Hence $C_{G}(D)$ has a block of defect $d$. Since $C_{G}(D)$ has a Sylow $p$-subgroup of $G$, by a theorem of R. Brauer $G$ has a block of defect $d$ (see Curtis-Reiner [3], Theorem (88.3) and Theorem (88. 8)). This is impossible.

Now we shall prove our theorem. Since a defect group of any 2-block of $G$ contains $\mathrm{O}_{2}(G)$, "if" part of the theorem is trivial.

We shall prove "only if" part of Theorem 1 by induction on the order of $G$. Suppose that "only if" part of Theorem 1 is not true. Let $G$ be a minimal counter example.

Lemma 3. The centralizer $C_{G}(x)$ of any non-identity 2-element $x$ of $G$ is a proper subgroup of $G$.

Proof. Suppose that $G=C_{G}(x)$ for some 2-element $x \neq 1$. Then by a theorem of R. Brauer ([1], p. 155), the blocks of $G$ and the blocks of $G /\langle x\rangle$ have one to one correspondence. Therefore every 2-block of $G /\langle x\rangle$ has defect $a^{\prime}$, where $|G /<x>|=2^{a \prime} \cdot g^{\prime},\left(2, g^{\prime}\right)=1$. By induction a Sylow 2-subgroup of $G /\langle x\rangle$ is normal. Hence a Sylow 2-subgroup of $G$ is 
normal, which is a contradiction. Hence $G>C_{G}(x)$ for every non-identity 2-element $x$ of $G$.

Lemma 4. The Sylow 2-subgroups of $G$ are indenpendent.

Proof. Let $x$ be any non-identity 2-element of $G$. Then by Lemma 2, Lemma 3 and by induction, a Sylow 2-subgroup of $C_{G}(x)$ is normal in $C_{G}(x)$. If two Sylow 2-subgroups $S_{1}, S_{2}$ of $G$ intersect non trivially, then the contralizer $C_{G}(x)$ of any non-identity element $x$ of $S_{1} \cap S_{2}$ contains $S_{1}$ and $S_{2}$. Since $C_{G}(x)$ is 2-closed, $S_{1}=S_{2}$. Hence the Sylow 2-subgroups of $G$ are independent.

By Lemma 1 and by a fundamental theorem of M. Suzuki [8], $G$ has a series of normal subgroups

$$
G \supset G_{1}>G_{2} \supset 1
$$

such that $G / G_{1}$ and $G_{2}$ are groups of odd order and $G_{1} / G_{2}$ is isomorphic to $L F\left(2,2^{a}\right)$. Put $\bar{G}=G / G_{2}$ and $\bar{L}=G_{1} / G_{2}$. We shall show that $\bar{G}$ has a irreducible character $X$ whose degree is divisible by $2^{a}$. As is well known, the group $\bar{L} \cong L F\left(2,2^{a}\right)$ has a irreducible character $\zeta$ of degree $2^{a}$. Let $X$ be a irreducible component of the induced character $\zeta^{\bar{G}}$. Then by the Frobenius' reciprocity theorem $\left.X\right|_{\bar{L}}$ contains $\zeta$ in its irreducible components. By a Clifford's theorem, $\left.X\right|_{\bar{L}}=e \sum_{g} \zeta^{g}$, where $e$ is an integer and the summation ranges over all the distinct conjugate character of $\zeta$. Hence $X(1) \equiv 0\left(\bmod 2^{a}\right)$. If we consider $X$ as a character of $G, X$ is a character of defect $O$. This contradicts with the assumption of Theorem 1. Thus we have proved "only if" part of our theorem. The proof is complete.

For an odd prime $p$, the following result of N. Ito [7] is known.

THEOREM 2. Let $G$ be a finite group all of whose $p$-blocks have the highest defects. If $G$ has a permutation representation of degree $p$, then a Sylow p-subgroup of $G$ is normal.

Actually in [7], N. Ito has proved a theorem different from Theorem 2. But considering his theorem from our point of view, we get easily Theorem 2.

The author thanks to Prof. N. Ito who has suggested him the formulation of Theorem 1. Our original one is Corollary to Theorem 1. 


\section{REFERENCES}

1] R. Brauer: Some applications of the theory of blocks of characters of finite groups I, J. of Algebra, Vol. 1 (1964), pp. 152-167.

2] N. Burgoyne and P. Fong: The Schur multipliers of the Mathieu groups, Nagoya Math. J., Vol. 27 (1966), pp. 733-745.

3 ] C. Curtis and I. Reiner: Representation theory of finite groups and associtaive algebras, New York, Interscience, 1962.

4] P. Fong: On the characters of p-solvable groups, Trans. Amer. Math. Soc. Vol. 98 (1961), pp. 263-284.

5] G. Frobenius: Über die Charaktere der mehrfach transitive Gruppen, Sitz. Preuss. Akad. Wissen. (1904), pp. 558-571.

6 ] P. Hall and G. Higman: On the p-length of p-soluble groups and reduction theorems for Burnside's problem, Proc. London Math. Soc. Vol. 6 (1956), pp. 1-42.

$7]$ N. Ito: Über die Darstellungen der Permutationsgruppen von Primzahlgrad, Math. Zeitschr. Vol. 89 (1965), pp. 196-198.

8 ] M. Suzuki: Finite groups of even order in which Sylow 2-groups are independent, Ann. of Math., Vol. 80 (1964), pp. 58-77.

Nagoya University 